\title{
Activation maps of convolutional neural networks as a tool for brain degeneration tracking in early diagnosis of dementia in Parkinson's disease based on magnetic resonance imaging
}

\section{Aleksandra Suwalska}

Silesian University of Technology: Politechnika Slaska

Joanna Siuda

Medical University of Silesia: Slaski Uniwersytet Medyczny w Katowicach

Szymon Kocot

Silesian University of Technology

Weronika Zmuda

Medical University of Silesia, Department of Neurology

Monika Rudzinska-Bar

Andrzej Frycz Modrzewski Krakow University, Department of Neurology

Joanna Polanska ( $\square$ joanna.polanska@polsı.pl)

Silesian University of Technology https://orcid.org/0000-0001-8004-9864

\section{Research note}

Keywords: Parkinson's disease, dementia, image biomarkers, deep learning, feature engineering, MRI

Posted Date: March 31st, 2021

DOI: https://doi.org/10.21203/rs.3.rs-362850/v1

License: () (1) This work is licensed under a Creative Commons Attribution 4.0 International License. Read Full License 


\section{Abstract}

Objective: Identification of Parkinson's Disease (PD) patients at risk for development of dementia is crucial for early intervention. Today, advanced, time-consuming and expensive imaging techniques (such as DaTSCAN-SPECT) are used for diagnosis. The study aimed to prove the usefulness of convolutional neural networks (CNN) for the identification of brain areas related to the progress of cognitive impairment by using standard Magnetic Resonance Imaging (MRI) sequences.

Results: T1\&T2 sequences of 18 patients were used in the pilot study. The activation maps were generated and the brain regions most involved in the classification process were identified that showed areas potentially significant in the diagnosis of cognitive impairment severity. The cerebellum was proven significant for distinguishing the above-mentioned classes in relative cerebellum volume (ANOVA $p$ value $=0.0038$ with large effect size $\eta 2=0.5254$ ) and folding ( $p$-value $=0.0031, \eta 2=0.5357$ ) which is consistent with reports by other authors. Our analysis demonstrated that the convolutional neural networks combined with the proper image preprocessing pipeline could be used for feature extraction in MRI sequences and can successfully support the identification of the disease-specific abnormalities of the brain image.

\section{Introduction}

Parkinson's disease (PD) is the second most common neurodegenerative disease which manifests cardinal motor symptoms of resting tremor, rigidity, bradykinesia and loss of postural stability [1]. The disease may also include a variety of non-motor symptoms, such as cognitive impairment, depression, sleep disturbances and autonomic dysfunction that affect patients' quality of life [2].

During the early stages, cognitive functions are impaired in about $40 \%$ of PD patients and almost $80 \%$ will develop dementia in the course of the disease [3]. Cognitive impairment may range from mild deficits up to dementia [4]. Early identification of PD patients at risk for development of dementia is crucial for assuring patients' well-being and proper medical intervention. Therefore, searching for easily accessible biomarkers is an important endeavour.

Neuroimaging investigations of cognitive impairment in PD is a topic of growing interest. Structural magnetic resonance imaging (MRI) studies have already established that patients who have Parkinson's disease with dementia (PDD) have atrophy of the parietal-temporal lobes, entorhinal cortex, hippocampus, prefrontal cortex and posterior cingulate-unlike PD patients without dementia or healthy controls $[5,6]$. Recently, research of new methods for improving the performance of MR image classification has started to be applied for medical purposes [7].

Many medical research problems are solved with the use of deep learning. Examples of applications include automatic segmentation of brain tumours [8], brain anatomical structures [9] and lesions [10], as well as the diagnosis of some neurological disorders such as Alzheimer's disease [11]. These methods have also found their application in the evaluation of Parkinson's disease symptoms, handwritten tests [12] 
and FP-CIT SPECT for dopamine transporter imaging [13] from which data is then used for training and classification by a deep neural network. The obtained class activation maps can provide information that actively supports the identification of parts of MR images that play a crucial role during the decision making process.

The study aimed to demonstrate that the developed convolutional neural network may serve as a tool in feature engineering, allowing identification of the damaged brain areas visualised by MRI in PD patients suffering from varying severities of cognitive impairment.

\subsection{Materials And Methods}

Twenty-one successive patients with previously diagnosed Parkinson's disease who reported to the outpatient clinic at the Medical University of Silesia, Katowice, were recruited for the prospective study. Written informed consent was obtained from all the participants. Finally, 18 PD patients without cognitive complaints or with differing severity of cognitive impairment constructed the patient groups. The demographic and clinical data of the patients are presented in Table 1. The subgroup of nine age-matched patients ( 3 persons per each diagnosis status) was additionally chosen from the initial 21 patients to inspect the identified biomarkers with no presence of the confounding factor. These patients were agematched with the use of a k-nearest neighbour algorithm-with the PD patients with mild cognitive impairment treated as the reference group (tests for mean value equality: patient age at the exam $p=0.1275$, age at the onset $p=0.2593$, disease duration $p=0.9746)$. PD was diagnosed according to the UK PD Society Brain Bank criteria (UKPDSBB) [14].

\section{Clinical assessment}

All subjects enrolled in the conducted study were carefully examined by a specialist and were subject to the Hoehn-Yahr test to estimate the stage of Parkinson's Disease. All the additional data such as disease onset, duration and information about the treatment were collected in a specially prepared survey. All patients were subject to the complex testing scenario, including neuropsychological test, Mini-Mental State Examination, Clock Drawing Test and Beck Depression Inventory. Other tests (Rey Auditory Verbal Learning Test, forward \& backward Digit Span subscale of Wechsler Adult Intelligence Scale, Trail Making Test (TMT parts A\&B) and Benton Visual Recognition Test (BVRT), were performed to estimate the strength of subjects cognitive.

Based on the results of the neuropsychological assessment, patients were then divided into three groups: cognitively normal (PD-CN), PD with mild cognitive impairment (PD-MCl) and PD with dementia (PDD). Cognitive impairment ( $\mathrm{MCl}$ or dementia) was diagnosed according to Movement Disease Society criteria $[4,15]$.

\section{MRI acquisition}

MRI scans were obtained using a General Electric 1.5 Tesla system. T1 and T2 weighted sequences were acquired for each patient. The total number of 2D scans acquired in the group of 18 patients was 5,984, for 
an average of 332.5 2D scans per patient.

\section{Data preprocessing and statistical analysis}

The MRI data were subject to a complex preprocessing pipeline. As a first step, data with visible crosstalk artefacts/slice overlap artefacts were identified and corrected. The median of Hodges-Lehman estimates of pairwise between-slice MRI signal intensity difference was calculated for each MRI sequence per each 3D study, and the affected 3D studies were identified with the use of Tukey's criterion. The next steps were (1) correction of magnetic field inhomogeneity, performed with the use of N4ITK technique [16]; (2) image intensity normalisation [17] to ensure the same signal range on each MRI sequence; and (3) brain extraction performed with an FSL-BET (Brain Extraction Tool) [18]. The images were downsized to a common resolution of $160 \times 160$ pixels. Additionally, the images were internally normalised with the use of a $z$-score algorithm. The final number of 2D scans used in the study was 5,760 .

The Shapiro-Wilk test was applied to verify the hypothesis of the distribution normality within each subgroup. Bartlett's test was used to check variance homogeneity. Depending on the test results, parametric ANOVA or nonparametric Kruskal-Wallis ANOVA was performed to test the hypothesis of mean/median value equality across all subgroups. Benjamini-Hochberg procedure was applied to correct for multiple testing. The post hoc Tukey-Kramer test was used in the pairwise comparisons. Effect size analysis [19] supported the findings obtained by standard statistical testing. Additionally, the above described statistical analysis was performed for the subgroup of the nine age-matched patients.

\section{Convolutional neural networks $(\underline{\mathrm{CNN}})$ model}

MR T1 and T2-weighted modalities were used to train the network.

The applied CNN consisted of 4 convolutional layers (kernel size of $3 \times 3$ pixels), followed by a max-pooling (kernel size of $2 \times 2$ pixels, a stride of 2) and batch normalisation modules. Each hidden layer was activated with the use of ReLU. Additionally, an image augmentation routine, including various image transformations such as rotation, rescaling, and shifting, was applied, which doubled the training set to $11,5202 \mathrm{D}$ scans. Nadam was used as the optimiser with a learning rate of 0.001 . The simplified structure of the proposed network is shown in Figure 1.

The CNN model was built and trained to estimate parameters used in the process of creating activation maps of the neuronal layers' classification. The CNN training was performed independently in three oneversus-other classification scenarios. The patient-level leave-one-out cross-validation schema was applied as presented in Figure 2. Gradient-weighted class activation maps [20,21], rescaled to the targeted resolution, were constructed for each patient, and the regions with the highest average across all patients activation values were recognised.

The segmentation of the identified distinguishing brain area was performed with the use of MiMSeg algorithm [22]. The segmented area relative volume and folding were estimated, and the basic descriptive statistics within each patient group were calculated. Similar to the statistical analysis of the clinical 
parameters, the hypothesis of mean value equality was verified by ANOVA test after checking if the test assumptions were upheld. Post hoc pairwise comparisons were performed to seek for group differences. A p-value of less than 0.05 was assumed as statistically significant.

\subsection{Result}

The severity of cognitive impairment could be assessed based on significant brain areas that are identified by visualising activation maps for a given disease entity. Regions of the highest importance were found in the frontal, posterior, temporal and cingulate cortex, gyrus lingualis, cerebellum, caudate nucleus, and thalamus; the exemplary maps are shown in Additional File 1, 2 and 3.

The cerebellum was chosen as an example for further detailed analysis due to its showing the highest importance in the automated diagnosis performed by the trained convolutional neural network. The average activation maps of the cerebellum for the three patient groups are presented in Additional File 4. The cerebella were automatically segmented with the use of MiMSeg algorithm. Cerebellum volume and folding were calculated and compared across the patient groups to verify the hypothesis of cerebellum importance in the context of dementia development. Their distributions are shown in Additional File 5.

The hypothesis of among group mean values equality of relative cerebellum volume and folding was investigated with the use of ANOVA tests (Additional File 6). The statistically significant change in relative cerebellum volume (Kruskal-Wallis ANOVA $p$-value $=0.0176)$ and cerebellum folding ( $p$-value $=0.0057$ ) of large effect size [23] were identified among the groups of patients showing volume and folding decrease with the progress of dementia. The findings remain significant in the subgroup of age-matched patients ( $p$ $=0.0273$ in both cases), even with a much larger effect size.

\subsection{Limitations}

It is well documented that a grey matter volume loss and cortical Lewy bodies pathology are involved in the development of cognitive decline in Parkinson's disease [24]. In cross-sectional studies about Parkinson's disease dementia (PDD), greater atrophy was observed in the frontal and temporal lobes compared to controls $[25,26]$. Our studies are consistent with this study, identifying the most involved areas in cognitive decline within the same brain regions.

Widespread atrophic changes are especially present in the limbic/paralimbic area $[27,28]$. Abnormalities associated with cognitive impairment within the posterior cingulate cortex (PCC), which is a part of the limbic system, arise from neurodegenerative processes. Moreover, finding PDD patient's metabolic deficits in this region that is heavily connected to the entorhinal cortex strongly suggests the possible influence of Alzheimer' s-like pathology in PD dementia $[24,27,29]$. Our results have also shown strong differences in PCC between PD patients with normal cognition and PD patients with dementia.

These subcortical atrophic changes are consistent with pathologic studies of PD dementia $[26,28]$. The predominant pattern of left hemisphere cortical atrophy in PD was described by Classen et al., where the 
rates of left hemisphere cortical atrophy were also strongly correlated with disease duration [30]. Other reports demonstrate that left-lateralised atrophy and left predominance is found in different neurodegenerative disorders with dementia [31,32].

In our study, the strongest association between the severity of cognitive impairment and cortical atrophy was detected in the cerebellum. More recent pathological studies have shown that there is an alphasynuclein aggregation pathology present in the cerebellum [33].

\subsection{Conclusions}

Our analysis proved that convolutional neural networks could be successfully used for feature engineering, even in cases of small sample studies. The proposed method utilises only widely available basic MRI sequences and, after very carefully designed image preprocessing and normalisation, can detect changes for which more time-consuming and expensive medical tests (such as DTI-MRI or DaT-SCAN-SPECT) are currently being used.

\section{Limitations}

Some limitations of our study should be recognised. We recruited a relatively small group of PD patients. However, we obtained significant and strong results in all discussed brain regions, and our results are in agreement with related literature. Additionally, the classical p-values are supported by the sample-sizeindependent effect size estimates. Another limitation was the fact that our PDD group was the oldest, and the age at disease onset was also higher in this group. To limit the impact of these confounding factors, an additional validation on a smaller group of age-matched patients was performed which confirmed our findings. According to the clinical variability in PD, we can distinguish two types of the disease - one with the onset of old age, and another of younger age [34]. The groups are characterised by rapid and slower disease progression, respectively, and along with advanced age, the risk of developing dementia in PD patients increases to $80 \%$ [35]. However, usually ageing results in diffuse, not focal cortical thinning [36], and our results showed a specific pattern of focal cortical and subcortical atrophy referring strictly to cognitive impairment assuming that age was not a confounding factor.

\section{Abbreviations}

AVLT - Auditory Verbal Learning Test

BDI - Beck Depression Inventory

BH FDR - Benjamini-Hochberg False Discovery Rate

BVRT - Benton Visual Recognition Test

CDT - Clock Drawing Test 
CNN - Convolutional Neural Network

LEDD - levodopa equivalent daily dose

$\mathrm{MCl}$ - Mild Cognitive Impairment

MMSE - Mini-Mental State Examination

MRI - Magnetic Resonance Imaging

PCC - Posterior Cingulate Cortex

PD - Parkinson's Disease

PD-CN - cognitively normal Parkinson's disease

PDD - Parkinson's Disease Dementia

PD-MCl - Parkinson's disease with Mild Cognitive Impairment

ReLU - Rectified Linear Unit

TMT - Trail Making Test

UKPDSBB - UK PD Society Brain Bank

UPDRS - Unified Parkinson's Disease Rating Scale

\section{Declarations}

Ethics approval and consent to participate: The study was approved by Ethics Committee of Medical University of Silesia (KNW/0022/KB1/112/16, dated 27.09.2016). Written informed consent was obtained from all the participants before the study initialisation.

Consent for publication: Not applicable.

Availability of data and materials: The datasets analysed during the current study are not publicly available but are available from the corresponding author on request.

Competing Interest: The authors declare no conflict of interest. The funders had no role in the design of the study; in the collection, analyses, or interpretation of data; in the writing of the manuscript, or in the decision to publish the results.

Funding: The study was partially financed by SUT/RAU4/SUBB-21 \& KNE-172/N/6/K projects. All calculations were carried out using GeCONil infrastructure funded by NCBiR project no. POIG.02.03.01-24099/13. 
Authors' Contributions: Conceptualization, Monika Rudzińska-Bar and Joanna Polanska; Data curation, Aleksandra Suwalska and Szymon Kocot; Formal analysis, Aleksandra Suwalska and Szymon Kocot; Funding acquisition, Joanna Siuda and Joanna Polanska; Methodology, Aleksandra Suwalska, Szymon Kocot and Joanna Polanska; Project administration, Joanna Siuda; Resources, Joanna Siuda and Weronika Żmuda; Software, Aleksandra Suwalska and Szymon Kocot; Validation, Joanna Siuda and Weronika Żmuda; Visualization, Aleksandra Suwalska and Szymon Kocot; Writing - original draft, Aleksandra Suwalska; Writing - review \& editing, Joanna Siuda, Monika Rudzińska-Bar and Joanna Polanska. All authors have read and agreed to the published version of the manuscript.

Acknowledgements: In memory of a good friend, we would like to thank and dedicate this work to $\mathrm{Dr}$ Franciszek Binczyk, whose great contribution and support made it possible to conduct the research and publish the results.

\section{References}

1. Postuma, RB; Berg, D; Stern, M; Poewe, W; Olanow, CW; Oertel, W; Obeso, J; Marek, K; Litvan, I; Lang, AE; Halliday, G; Goetz, CG; Gasser, T; Dubois, B; Chan, P; Bloem, BR; Adler, CH; Deuschl, G. MDS clinical diagnostic criteria for Parkinson's disease. Mov Disord. 2015; 30(12), 1591-601.

2. Fereshtehnejad SM, Zeighami Y, Dagher A, Postuma RB. Clinical criteria for subtyping Parkinson's disease: biomarkers and longitudinal progression. Brain. 2017;140(7):1959-1976.

3. Anang JB, Gagnon JF, Bertrand JA, Romenets SR, Latreille V, Panisset M, Montplaisir J, Postuma RB. Predictors of dementia in Parkinson disease: a prospective cohort study. Neurology. 2014;83(14):125360.

4. Emre M, Aarsland D, Brown R, Burn DJ, Duyckaerts C, Mizuno Y, Broe GA, Cummings J, Dickson DW, Gauthier S, Goldman J, Goetz C, Korczyn A, Lees A, Levy R, Litvan I, McKeith I, Olanow W, Poewe W, Quinn N, Sampaio C, Tolosa E, Dubois B. Clinical diagnostic criteria for dementia associated with Parkinson's disease. Mov Disord. 2007;22(12):1689-707.

5. Tuite PJ, Mangia S, Michaeli S. Magnetic Resonance Imaging (MRI) in Parkinson's Disease. J Alzheimers Dis Parkinsonism. 2013; Suppl1. doi:10.4172/2161-0460.S1-001.

6. Kandiah N, Mak E et al. Cerebral white matter hyperintensity in Parkinson's disease: A major risk factor for mild cognitive impairment. Parkinsonism and Related Disorders 2013;19: 680-683.

7. Miyake J., Ohigashi H., Niioka H., Kaechang P. Medical Application of Artificial Intelligence/Deep Learning. Brain and nerve Shinkei kenkyu no shinpo, 2009 71(1), 5-14.

8. Isensee F., Kickingereder P., Wick W., Bendszus M., Maier-Hein, K. H. (2017, September). Brain Tumor Segmentation and Radiomics Survival Prediction: Contribution to the BRATS 2017 Challenge. In International MICCAI Brainlesion Workshop (pp. 287-297). Springer, Cham.

9. de Brebisson A and Montana G (2015). Deep neural networks for anatomical brain segmentation. Proceedings of the IEEE Conference on Computer Vision and Pattern Recognition Workshops. 2015.

10. Kamnitsas K, Ledig C, Newcombe V, Simpson J, Kane A, Menon D, Glocker B. (2017). Efficient multiscale 3D CNN with fully connected CRF for accurate brain lesion segmentation. Medical image 
analysis, 36, 61-78.

11. Wang S, Phillips P, Sui Y, Liu B, Yang M,Cheng H. (2018). Classification of Alzheimer's Disease Based on Eight-Layer Convolutional Neural Network with Leaky Rectified Linear Unit and Max Pooling. Journal of medical systems, 42(5), 85.

12. Pereira CR, Pereira D, Papa J, Rosa G, Yang X. (2016). Convolutional neural networks applied for parkinson's disease identification. In Machine Learning for Health Informatics (pp. 377-390). Springer, Cham.

13. Choi H, Ha S, Im H, Paek S, Lee D. S. (2017). Refining diagnosis of Parkinson's disease with deep learning-based interpretation of dopamine transporter imaging. Neurolmage: Clinical, 16, 586-594.

14. Hughes AJ, Daniel SE, Kilford L, Lees AJ. Accuracy of clinical diagnosis of idiopathic Parkinson's disease. A clinico-pathological study of 100 cases. JNNP 1992;55:181-184.

15. Litvan I, Aarsland D, Adler CH, Goldman JG, Kulisevsky J, Mollenhauer B, Rodriguez-Oroz MC, Tröster Al, Weintraub D. MDS Task Force on mild cognitive impairment in Parkinson's disease: critical review of PD-MCl. Mov Disord. 2011;26(10):1814-24.

16. Tustison NJ, Avants BB, Cook PA, Zheng Y, Egan A, Yushkevich PA, Gee JC. N4ITK: improved N3 bias correction. IEEE transactions on medical imaging, 29(6),2010 1310-1320.

17. Nyúl, L. G., Udupa, J. K., Zhang, X. (2000). New variants of a method of MRI scale standardisation. IEEE transactions on medical imaging, 19(2), 143-150.

18. Smith SM. Fast robust automated brain extraction. Human Brain Mapping, 17(3):143-155, November 2002.

19. Cohen J. Statistical power analysis for the behavioral sciences. 2nd ed. Lawrence Erlbaum Associates, Publisher; 1988.

20. Selvaraju R, Cogswell M, Das A, Vedantam R, Parikh D, Batra D. (2017, October). Grad-cam: Visual explanations from deep networks via gradient-based localisation. In 2017 IEEE International Conference on Computer Vision (ICCV) (pp. 618-626). IEEE.

21. Petersen R, Aisen P, Beckett L, Donohue M, Gamst A, Harvey D, Trojanowski, J. (2010). Alzheimer's disease neuroimaging initiative (ADNI): clinical characterisation. Neurology, 74(3), 201-209.

22. Binczyk F, Stjelties B, Weber C, Goetz M, Meier-Hein K, Meinzer HP, Polanska, J. (2017). MiMSeg-an algorithm for automated detection of tumor tissue on NMR apparent diffusion coefficient maps. Information Sciences, 384, 235-248.

23. Cohen BH. Explaining psychological statistics. 3rd ed. New York: John Wiley \& Sons; 2008.

24. Hwanga KS, Beyer MK, Green AE, Chung C, Thompson PM, Janving C, Larsen JP, Aarsland D, Apostolova LG. Mapping Cortical Atrophy in Parkinson's Disease Patients with Dementia. J Parkinsons Dis. $2013 ; 3(1)$ : 69-76.

25. Silbert LC, Kaye JA. Neuroimaging and Cognition in Parkinson's Disease Dementia. Brain Pathol. 2010; 20(3):646-653.

26. Burton EJ, McKeith JG, Burn DJ, Williams ED, O'Brien JT. Cerebral atrophy in Parkinson's disease with and without dementia: a comparison with Alzheimer's disease, dementia with Lewy bodies and 
controls. Brain 2004; 127: 791-800.

27. Leech R, Sharp DJ. The role of the posterior cingulate cortex in cognition and disease. Brain 2014:137; 12-32.

28. Nagano-Saito, Washimi Y, Arahata Y, Kachi T,Lerch JP, Evans AC, Dagher A, Ito K. Cerebral atrophy and its relation to cognitive impairment in Parkinson disease. Neurology 2005;64:224 - 229.

29. Camicioli RM, Korzanb JR, Fostera SL, Fisherd NJ, Emery DJ, Bastos AC, Hanstock CC. Posterior cingulate metabolic changes occur in Parkinson's disease patients without dementia. Neuroscience Letters 2004; 354: 177-180.

30. Claassen DO, McDonell KE, Donahue M, Rawal S, Wylie SA, Neimat JS, Kang H, Hedera P, Zald D, Landman B, Dawant B, Rane S. Cortical asymmetry in Parkinson's disease: early susceptibility of the left hemisphere. Brain Behav. 2016;6(12):e00573.

31. Bruck, A. Hippocampal and prefrontal atrophy in patients with early non-demented Parkinson's disease is related to cognitive impairment. Journal of Neurology, Neurosurgery \& Psychiatry, 2004;75:14671469.

32. Mak, E., Zhou, J., Tan, L. C., Au, W. L., Sitoh, Y. Y., \& Kandiah, N. Cognitive deficits in mild Parkinson's disease are associated with distinct areas of grey matter atrophy. Journal of Neurology, Neurosurgery and Psychiatry 2014:85:576-580.

33. Seidel K, Bouzrou M, Heidemann N, Kruger R, Schols L, den Dunnen WFA, Korf HW, Rub U. Involvement of the Cerebellum in Parkinson Disease and Dementia with Lewy Bodies. Ann Neurol 2017;81:898903.

34. Van Rooden SM, Colas F, Martinez-Martin P, et al. Clinical subtypes of Parkinson's disease. Mov Disord $2011 ; 26(1): 51-58$.

35. Xu Y, Jing Yang J, Shang H. Meta-analysis of risk factors for Parkinson's disease dementia. Translational Neurodegeneration 2016;5:11.

Hurtz S, Woo E, Kebets V, Green AE, Zoumalan C, Wang B, Ringman JM, Thompson PM, Apostolova LG. Age Effects on Cortical Thickness in Cognitively Normal Elderly Individuals. Dement Geriatr Cogn Disord Extra $2014 ; 4: 221-227$

\section{Tables}

Table 1. Demographic and clinical data of the studied group (given as mean value \pm standard deviation). 


\begin{tabular}{|c|c|c|c|c|c|c|}
\hline Parameter & $\begin{array}{l}\text { PD } \\
\text { (all patients) }\end{array}$ & $\begin{array}{l}\text { PD-CN } \\
\text { (cognitively } \\
\text { normal) }\end{array}$ & $\begin{array}{l}\text { PD-MCl } \\
\text { (mild } \\
\text { cognitive } \\
\text { impairment) }\end{array}$ & $\begin{array}{l}\text { PDD } \\
\text { (with } \\
\text { dementia) }\end{array}$ & $\begin{array}{l}\text { Unadjusted } \\
\text { ANOVA } \\
\text { P-value }\end{array}$ & $\begin{array}{l}\text { BH FDR- } \\
\text { adjusted } \\
\text { ANOVA } \\
\text { P-value }\end{array}$ \\
\hline No & 18 & 10 & 4 & 4 & & \\
\hline $\mathrm{F} / \mathrm{M}$ & $3 / 15$ & $3 / 7$ & $0 / 4$ & $0 / 4$ & & \\
\hline Age at onset & $57.50 \pm 9.13$ & $55.20 \pm 7.10$ & $53.00 \pm 8.29$ & $\begin{array}{l}67.75 \pm \\
8.22\end{array}$ & $0.024183^{A}$ & 0.043721 \\
\hline $\begin{array}{l}\text { Age at } \\
\text { examination }\end{array}$ & $66.22 \pm 8.50$ & $63.60 \pm 5.21$ & $61.50 \pm 8.81$ & $\begin{array}{l}77.50 \pm \\
5.26\end{array}$ & $0.002800^{A}$ & 0.016853 \\
\hline $\begin{array}{l}\text { Disease } \\
\text { duration }\end{array}$ & $8.78 \pm 4.71$ & $8.50 \pm 4.12$ & $8.50 \pm 5.20$ & $\begin{array}{l}9.75 \pm \\
6.80\end{array}$ & $0.907278^{A}$ & 0.907278 \\
\hline $\begin{array}{l}\text { Hoehn-Yahr } \\
\text { stage }\end{array}$ & $2.44 \pm 0.62$ & $2.20 \pm 0.42$ & $2.50 \pm 1.00$ & $3.0 \pm 0.0$ & $0.044403^{\mathrm{B}}$ & 0.062904 \\
\hline $\begin{array}{l}\text { UPDRS III } \\
\text { ON }\end{array}$ & $\begin{array}{l}17.00 \pm \\
10.14\end{array}$ & $12.50 \pm 8.37$ & $21.00 \pm 12.27$ & $\begin{array}{l}24.25 \pm \\
7.95\end{array}$ & $0.091764^{\mathrm{A}}$ & 0.119999 \\
\hline $\begin{array}{l}\text { UPDRS III } \\
\text { OFF }\end{array}$ & $35.67 \pm 14.51$ & $29.80 \pm 11.9$ & $45.00 \pm 18.60$ & $\begin{array}{l}41.0 \pm \\
12.38\end{array}$ & $0.147291^{A}$ & 0.178853 \\
\hline $\begin{array}{l}\text { Dominant } \\
\text { side }(R / L)\end{array}$ & $12 / 6$ & $8 / 2$ & $3 / 1$ & $1 / 3$ & $0.177117^{C}$ & 0.200733 \\
\hline LEDD & $806.4 \pm 372.6$ & $698.5 \pm 309.3$ & $1235.0 \pm 264.4$ & $\begin{array}{l}647.5 \pm \\
335.2\end{array}$ & $0.020762^{A}$ & 0.043721 \\
\hline MMSE & $26.28 \pm 3.89$ & $28.20 \pm 1.14$ & $26.75 \pm 1.50$ & $\begin{array}{l}21.00 \pm \\
5.48\end{array}$ & $0.042451^{B}$ & 0.062904 \\
\hline CDT & $7.72 \pm 3.08$ & $9.80 \pm 0.63$ & $7.00 \pm 2.94$ & $\begin{array}{l}3.25 \pm \\
1.50\end{array}$ & $0.001709^{B}$ & 0.016852 \\
\hline BDI & $9.78 \pm 5.85$ & $9.70 \pm 5.93$ & $12.75 \pm 6.65$ & $\begin{array}{l}7.00 \pm \\
4.69\end{array}$ & $0.403331^{A}$ & 0.428539 \\
\hline $\begin{array}{l}\text { AVLT } \\
\text { delayed } \\
\text { recall }\end{array}$ & $5.22 \pm 3.81$ & $7.40 \pm 3.37$ & $3.75 \pm 2.63$ & $\begin{array}{l}1.25 \pm \\
1.26\end{array}$ & $0.007159^{A}$ & 0.020284 \\
\hline $\begin{array}{l}\text { BVRT } \\
\text { mistakes }\end{array}$ & $5.22 \pm 2.92$ & $4.20 \pm 2.04$ & $4.25 \pm 2.50$ & $\begin{array}{l}8.75 \pm \\
2.87\end{array}$ & $0.025718^{B}$ & 0.043721 \\
\hline $\begin{array}{l}\text { Verbal } \\
\text { fluency }\end{array}$ & $11.78 \pm 2.94$ & $13.60 \pm 1.65$ & $11.00 \pm 2.00$ & $\begin{array}{l}8.00 \pm \\
2.45\end{array}$ & $0.002974^{B}$ & 0.016853 \\
\hline $\begin{array}{l}\text { Semantic } \\
\text { fluency }\end{array}$ & $14.89 \pm 3.79$ & $17.20 \pm 2.30$ & $14.25 \pm 2.99$ & $\begin{array}{l}9.75 \pm \\
1.71\end{array}$ & $0.006779^{B}$ & 0.020284 \\
\hline $\begin{array}{l}\text { Digit span } \\
\text { forward }\end{array}$ & $5.67 \pm 1.14$ & $6.00 \pm 1.05$ & $6.25 \pm 0.50$ & $\begin{array}{l}4.25 \pm \\
0.50\end{array}$ & $0.025080^{B}$ & 0.043721 \\
\hline Digit span & $4.11 \pm 1.41$ & $4.90 \pm 0.88$ & $4.25 \pm 0.96$ & $2.00 \pm$ & $0.006734^{B}$ & 0.020284 \\
\hline
\end{tabular}


$\mathrm{F}=$ female, $\mathrm{M}$ = male, UPDRS = Unified Parkinson's disease rating scale, LEDD = levodopa equivalent daily dose, $\mathrm{MMSE}=$ Mini-Mental State Examination, $\mathrm{CDT}=$ Clock Drawing Test, BDI = Beck Depression Inventory, AVLT = Auditory Verbal Learning Test, BVRT = Benton Visual Recognition Test, BH FDR-adjusted = Benjamini-Hochberg False Discovery Rate adjusted A parametric ANOVA, B Kruskal-Wallis ANOVA, C Fisher exact test.

\section{Figures}

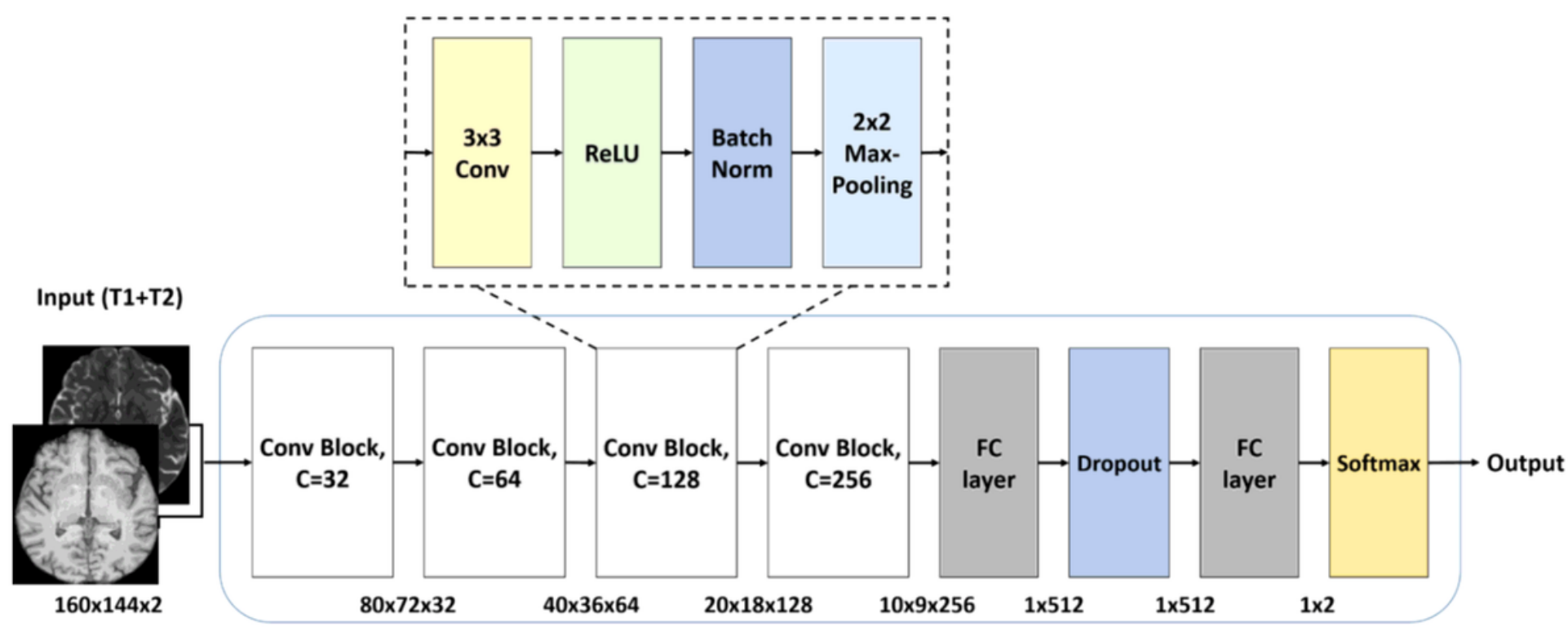

Figure 1

Structure of the proposed convolutional neural network.

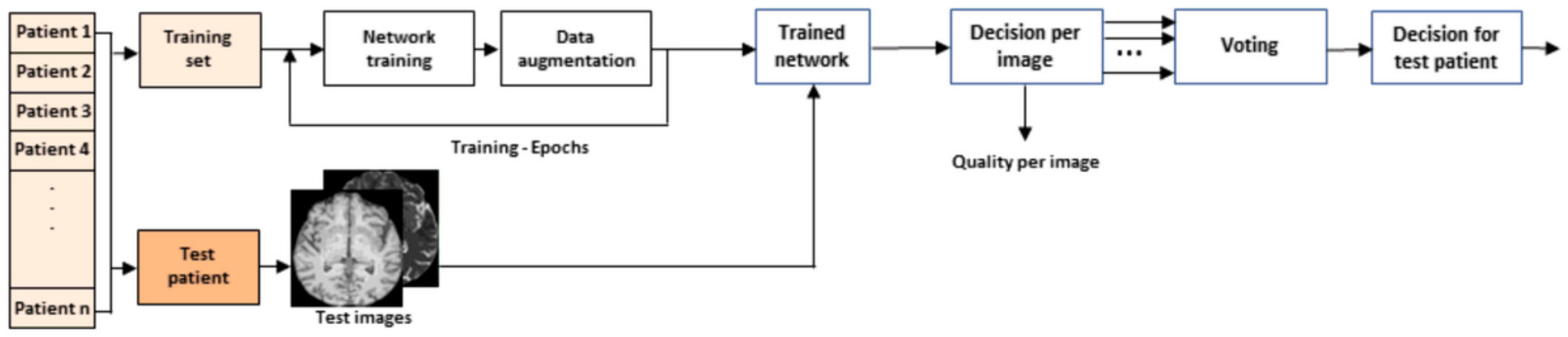

Figure 2

Diagram of a single loop of the Leave-One-Out validation experiment. The experiment was repeated for each patient independently. 


\section{Supplementary Files}

This is a list of supplementary files associated with this preprint. Click to download.

- AdditionalFile1.tiff

- AdditionalFile2.tiff

- AdditionalFile3.tiff

- AdditionalFile4.tiff

- AdditionalFile5.tiff

- AdditionalFile6.docx 